\title{
Tests for field mould and associated mycotoxins in South Australian lentil (Lens culinaris) grain
}

\author{
J. A. Davidson • L. S. McMurray • C. J. Wilmshurst • \\ S. A. Sherriff • A. M. Pointon
}

Received: 4 July 2011 / Accepted: 13 April 2012 / Published online: 29 April 2012

(C) Australasian Plant Pathology Society Inc. 2012

\begin{abstract}
Rain events in South Australia during the lentil harvest of 2010/2011 raised concerns as to whether grain had been infected with field moulds and associated mycotoxins. Grain samples from commercial crops were tested for the mycotoxins Deoxynivalenol (DON = Vomitoxin), Fumonisin B1 and B2 and zearalenone, which are produced by Fusarium spp. Sampling was biased towards more severely affected grain to increase the probability of detection of moulds and toxins, including worst case samples of discarded waste grain (screenings) from cleaning plants. No mycotoxins were detected although Fusarium spp. were detected in all but three of the samples. No Alternaria or Aspergillus spp. were detected. Fusarium spp. were detected at a higher level in grain harvested after a significant rain event in December 2010 compared to grain harvested prior to this event. Percentage of stained seed was higher in plants sampled in machinery wheel tracks compared to those from the remainder of the paddock. The fungal pathogen Ascochyta lentis was also detected on the grain but percentage infection was not affected by harvest date. No Botrytis spp. were detected on the samples. The pilot study identified that the lentil grain in these samples from the 2010/2011 harvest in South Australia was free of mycotoxins and posed no risk to humans or livestock.
\end{abstract}

Keywords Ascochyta lentis · Fusarium · Mycotoxin . Seed infection

J. A. Davidson $(\bowtie) \cdot$ C. J. Wilmshurst · A. M. Pointon South Australian Research and Development Institute, GPO Box 397, Adelaide, South Australia 5001, Australia e-mail: jenny.davidson@sa.gov.au

L. S. McMurray $\cdot$ S. A. Sherriff

South Australian Research and Development Institute,

PO Box 822, Clare, South Australia 5453, Australia
The South Australian lentil crop harvested in 2010/2011 experienced quality issues including weather and disease staining (Ascochyta lentis, Botrytis cinerea and or B. fabae) on the grain, unfilled grain and wrinkled seed coat. Rain events after crop maturity and pre-harvest of many crops also raised concerns amongst handlers and marketers that the grain was infected with field moulds (e.g. Alternaria spp., Aspergillus spp., Fusarium spp.). These in turn could produce mycotoxins in the grain detrimental to stock and humans. Fusarium spp. are known to produce the mycotoxins Deoxynivalenol $(\mathrm{DON}=$ Vomitoxin), Fumonisin B1 and B2 and zearalenone particularly in small cereal grains such as wheat and barley (Bottalico and Perrone 2002) while aflatoxins are produced by Aspergillus spp. particularly in peanuts and tree nuts (Cressey 2009). There is limited information about mycotoxins in grain legumes (pulses) grown for human consumption.

The National Residues Survey (NRS) has set levels of detection and quantification for Australian stockfeed at $<50 \mu \mathrm{g} / \mathrm{kg}$ for DON and Fumonisin B1 and B2, and $<10 \mu \mathrm{g} / \mathrm{kg}$ for zearalenone (DAFF 2011). Maximum levels of these mycotoxins in human food in the United States and European Union are set at $1-4 \mathrm{mg} / \mathrm{kg}$ and $0.5 \mathrm{mg} / \mathrm{kg}$, respectively (FAO 2004); being 100 times more than the Australian detection levels due to the low proportion of foods likely to be contaminated in the overall daily intake. No maximum levels for these mycotoxins have been set for human foods in Australia by Food Standards Australian New Zealand (FSANZ) as they have been assessed as posing a low public health risk. A series of risk assessments conducted by FSANZ lead to just three classes of mycotoxins included in the Food Standards Code; aflatoxins in peanut and tree nuts, ergot in cereal grains and phomopsins in lupin seeds and products of lupin seeds (Cressey 2009). 
The National Agricultural Commodities Marketing Association (NACMA) receival standards for lentil grain in Australia differ for the aforementioned traits in that Premium grade lentils have a $1 \%$ tolerance in a subsample for quality issues such as weather staining, disease staining, unfilled grain and wrinkled seed coat but zero tolerance across the entire load for field moulds (Pulse Australia 2011). The trading standards have been set at nil tolerance for field mould due to a nil tolerance in the marketplace brought about by visual concerns and the potential for food safety issues arising from the presence of field and or storage moulds. Some marketers however have an allowable out turn standard of $1 \%$ affected grain. Lentil grain is assessed for these quality traits at silos by a visual appraisal based on industry derived colour charts (Pulse Australia 2011). However subjective testing often cannot distinguish between field mould and the other traits such as ascochyta blight that cause seed staining. As some of these other traits do have allowable tolerance levels the potential exists for lentils to be rejected or downgraded on the basis of non-existent field mould.

A pilot survey was conducted to test for the presence of field moulds and associated hazardous mycotoxins in samples from the 2010/2011 harvest in South Australia rejected at receival or assessed as severely affected by quick visual examination. Samples tested were biased to those more severely affected to increase the probability of detection of moulds and toxins including worst case samples of discarded waste grain (screenings) from cleaning plants. Consequently, with this design a negative result would indicate an absence of any food safety risk and an opportunity to improve marketing potential (for example by seed cleaning) to remove what is an aesthetic problem.

A total of 24 lentil samples, each $2 \mathrm{~kg}$, representative of harvested grain was collected from farm storage (silos, sheds or grain bags) across the main lentil growing district in South Australia (Yorke Peninsula) and consisted of 16 samples of cv. Nugget, 4 of cv. Nipper, 2 of cv. Northfield and 2 of cv. PBA Flash. The disease resistance ratings of these cultivars are listed in Table 1. These samples each had less than $1 \%$ discoloured grain. A further four samples, each less than $500 \mathrm{~g}$, consisting entirely of selected stained and damaged seed which had been removed by a cleaning process, were also collected from grain handlers or farms.

Seed testing was conducted by a laboratory with National Association of Testing Authorities (NATA) accreditation for mycotoxins. A mould test counting the number of colony forming units (CFU) was conducted on each of the 24 representative samples and resulting fungal colonies were identified to genera. The presence of mycotoxins DON, Fumonisin B1 and B2 and zearalenone was tested for in all 28 samples at levels specified by the NRS.

The percentage incidence of $A$. lentis and Botrytis spp. was tested on the 24 representative samples at the SARDI Pulse Pathology laboratory in Adelaide using International Seed Testing Association (ISTA) procedures as follows. A randomly selected subset of 400 seeds from each sample was surface sterilised for 2 min with $2 \%$ sodium hypochlorite, air dried and transferred to potato dextrose agar plates. The plates were incubated at room temperature under ultra-violet and white light with $12 \mathrm{~h} / 12 \mathrm{~h}$ day/night cycles for 14 days. At the end of the incubation period the number of seeds with fungal cultures of $A$. lentis or Botrytis spp. was counted and converted to percentage infection for each sample.

The influence of agronomic practices on seed quality was also investigated in five paddocks cropped to lentils across the Yorke Peninsula and Mid North regions of South Australia. Prior to harvest but following a rain event on the mature crop, seeds were sampled from three positions selected at random from within wheel tracks and another three positions outside of wheel tracks, in each paddock, 200 seed per position. Seed samples were assessed for $\%$ staining and results analysed using analysis of variance.

Rainfall records showed that the major rain event during lentil harvest occurred on 8 December 2010 with $32 \mathrm{~mm}$ at Maitland (34.4 S, $137.7 \mathrm{E})$ and $52 \mathrm{~mm}$ at Kadina (34.0 S, 137.7 E) on Yorke Peninsula and $72 \mathrm{~mm}$ at Snowtown (33.8 $\mathrm{S}, 138.2^{\circ} \mathrm{E}$ ) in the Mid North region of South Australia. The long term median rainfall for all of December at these sites is

Table 1 Disease resistance ratings ${ }^{\mathrm{a}}$ and results of seed infection tests for lentil cultivars tested for fungal infection on seed and presence of associated mycotoxins

\begin{tabular}{|c|c|c|c|c|}
\hline \multirow[b]{2}{*}{ Cultivar } & \multicolumn{3}{|c|}{ Ascochyta blight } & \multirow[b]{2}{*}{ Botrytis grey mould } \\
\hline & Foliage & Seed & Range of $\%$ seed infection & \\
\hline PBA Flash & $\mathrm{MS}^{\mathrm{b}}$ & MS to MR & $4.0-8.5$ & $\mathrm{~S}$ \\
\hline Nipper & $\mathrm{R}$ & $\mathrm{R}$ & $0.5-5.0$ & $\mathrm{R}$ \\
\hline Nugget & MR & MS to MR & $0.0-12.5$ & MR \\
\hline Northfield & $\mathrm{R}$ & $\mathrm{R}$ & $0.0-0.25$ & $\mathrm{R}$ \\
\hline
\end{tabular}

${ }^{\text {a }}$ Resistance ratings from Hawthorne et al. 2011

${ }^{\mathrm{b}} R$ resistant, $M R$ moderately resistant, $M S$ moderately susceptible, $S$ susceptible 
Table 2 Number of colony forming units (CFU) of Fusarium spp. detected in 24 lentil grain samples from the 2010/2011 harvest in South Australia

\begin{tabular}{lllc}
\hline Cultivar & $\begin{array}{l}\text { Harvested pre or } \\
\text { post rain of } \\
\text { 8 December 2010 }\end{array}$ & $\begin{array}{l}\text { Number } \\
\text { of samples }\end{array}$ & CFU range \\
\hline Nugget & Pre rain & 5 & Undetected-3000 \\
& Post rain & 8 & $250-4699$ \\
& Unknown & 3 & Undetected-600 \\
Nipper & Pre rain & 0 & - \\
& Post rain & 3 & $200-2100$ \\
& Unknown & 1 & 300 \\
Northfield & Pre rain & 1 & 700 \\
& Post rain & 1 & 200 \\
Flash & Pre rain & 1 & Undetected \\
& Post rain & 1 & 4500 \\
Mean of & Pre rain & 7 & 1071 \\
samples & Post rain & 13 & 2200 \\
\hline
\end{tabular}

Means significantly different at $P<0.05$

$18.5 \mathrm{~mm}, 12.5 \mathrm{~mm}$ and $14.6 \mathrm{~mm}$, respectively. All other rain events during harvest (November and December of 2010) at these sites were $7 \mathrm{~mm}$ or less (Bureau of Meteorology 2011), however other localised major rainfall events were reported as occurring in these regions in November and December 2010. Fungal counts on grain samples harvested before and after the major rain event of 8 December 2010 were compared using ttests and mean separation was based on least significant differences at $5 \%$ level of probability. Cultivars resistant to A. lentis were not included in the analysis of ascochyta infection.

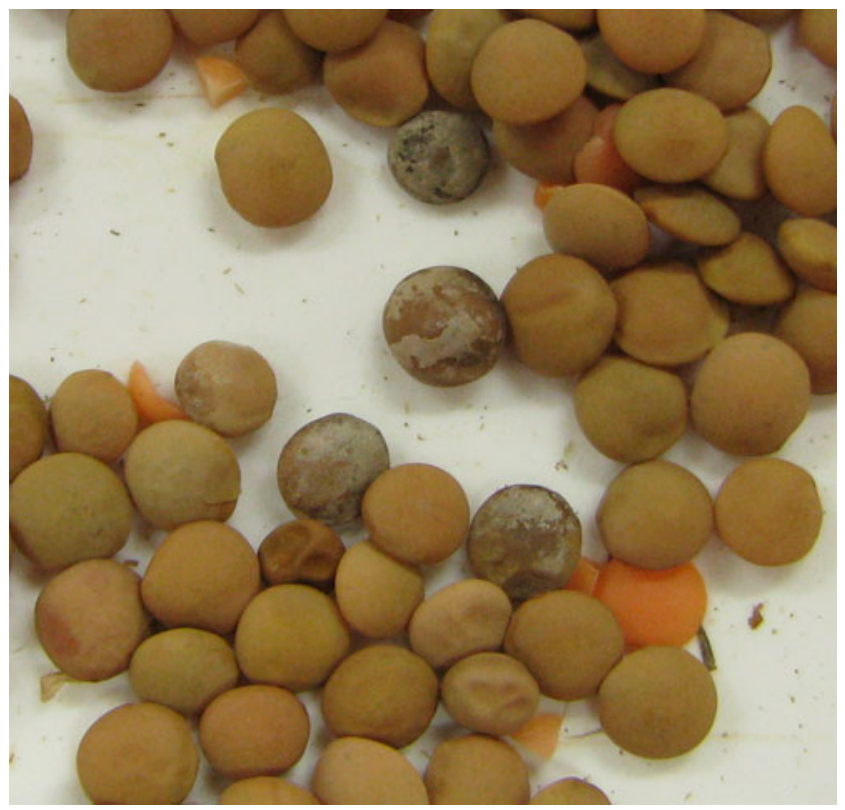

Fig. 1 Field mould on lentil grain harvested in South Australia in 2010/2011

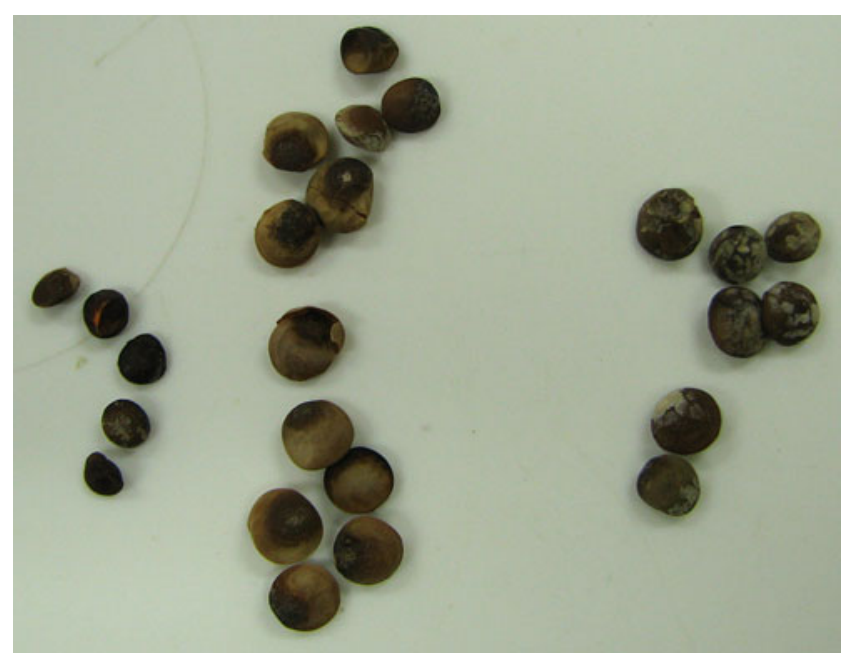

Fig. 2 Unfilled grain (Left), ascochyta stain (Centre), disease and or field mould (Right) on grain selected out of the 2010/2011 South Australian lentil harvest

No Fusarium toxins (DON, Fumonisin B1 and B2, Zearalenone) were detected in any of the 28 lentil grain samples from the South Australian 2010/2011 harvest tested in this study, including the four composites samples of defective grain. All detected moulds were of the Fusarium genera and were in all but three samples (two of which were known to be harvested before the major rain event) at varying levels on the grain (Table 2) while Aspergillus and Alternaria spp. were not detected in any samples. Grain samples harvested prior to the major rain event of 8 December 2010 had mould counts ranging from undetected in two samples to a maximum of 3,000 CFU/g. The grain samples harvested after the major rain event had mould counts ranging from 200 to a maximum of 4,699 CFU/g. The mean count post rain $(2,200 \mathrm{CFU})$ was significantly $(P<0.05)$ greater than the mean count $(1,071 \mathrm{CFU})$ for samples harvested pre December rain.

Table 3 Percentage of stained seed in samples collected from inside and outside of machinery wheel tracks in five mature lentil crops in South Australia 2010

\begin{tabular}{lccc}
\hline Crop Number & $\begin{array}{l}\text { \% stained seed } \\
\text { outside of } \\
\text { wheel tracks }\end{array}$ & $\begin{array}{l}\text { \% stained } \\
\text { seed inside } \\
\text { wheel tracks }\end{array}$ & $\begin{array}{l}\text { Mean \% } \\
\text { stained seed } \\
\text { per crop }\end{array}$ \\
\hline 1 & 1.67 & 4.83 & 3.25 \\
2 & 3.83 & 8.83 & 6.33 \\
3 & 0.50 & 2.00 & 1.25 \\
4 & 3.83 & 4.83 & 4.33 \\
5 & 1.67 & 4.83 & 3.25 \\
Mean \% stained & 2.30 & 5.07 & \\
seed in or out & & & \\
of wheel tracks & & & \\
\hline
\end{tabular}

L.S.D $(P<0.05)$ crops $=2.72$; L.S.D $(P<0.01)$ in or out of wheel tracks $=1.72$; interaction is not significant 
Ascochyta lentis infection levels on the 24 lentil samples ranged from undetected in five samples to a maximum of $12.5 \%$ (Table 1). Least infection was recorded in the resistant cvs Northfield and Nipper while the maximum infection rates were recorded in more susceptible cvs Nugget and Flash. There was no significant difference in ascochyta blight infection rates between samples harvested pre and post the rain on 8 December 2010. The recommendation for lentils is that seed with more than $5 \%$ infection poses a risk for an ascochyta blight epidemic (Hawthorne et al. 2011). There is no risk to humans or stock if ascochyta blight infected seed is consumed. No Botrytis spp. infection was detected on any of the samples despite this disease being identified in many lentil crops in South Australia during the 2010 growing season. Visual appraisal of seed for field mould (Fig. 1) and disease or weather damage were sometimes indistinguishable (Fig. 2).

Amongst the five crops sampled for stained seed in and out of machinery wheel tracks there was significant $(P<0.05)$ variation in percentage of stained seed, ranging from $1.3 \%$ to $6.3 \%$. Seed staining was significantly $(P<0.01)$ increased in the wheel tracks by an average of twice the amount in the remainder of the paddock (Table 3).

Mould counts detect all fungi on tested grain, including infection not apparent by visual inspection. Visible infection only occurs if sufficient moisture is present for fungal growth as demonstrated by higher counts following the December rain. As such due care must be taken not to overestimate the risk indicated by mould counts. The few studies conducted on lentil grain have found that Fusarium spp. are regularly found on lentil grain, ranging from $0.02 \%$ to $15 \%$ seed infected and a wide range of other fungal species have also been detected at similar levels (Attitalla et al. 2010; El-Maraghy 1988; El-Nagarabi and Elshafie 2000; Husssain et al. 2007; Rahim et al. 2010). These studies have been conducted on grain samples from farms and markets in Pakistan and north Africa, where grain storage facilities are most likely substandard to Australian conditions and grain would be exposed to variable environmental conditions. However similar fungal contamination has been detected on pulse grain in Canada (Sumar and Howard 1983) and in US Pacific Northwest (Kaiser 1992). One study suggested that lentil grain has a lower propensity for fungal infection compared to cereal grains and nuts (Attitalla et al. 2010).

The current study was not a systematic survey and was intentionally biased toward worst case samples of lentil grain from the 2010/2011 harvest in South Australia. Even under these extreme conditions, no evidence of mycotoxins was found in any sample, including seed specifically selected for a high degree of seed staining. Test results indicated that these samples posed no threat to human or stock safety. Fusarium spp. were detected in many of the samples. Fusarium spp. are a common fungal component of soils and it is probable that grain in the low growing lentil crop is regularly infected with this fungus. Further studies are required to investigate whether the risk of mycotoxin production by Fusarium spp. in lentils is low as indicated here.

Care must be taken in lentil production to deliver a high quality product to the discerning market. Agronomic management to minimise seed staining will also reduce the risk of mis-diagnosis of field mould. Timely harvest where possible, including harvesting before rain events on the mature crop, will reduce the percentage of stained seed. Seed staining was higher in wheel tracks than other parts of the paddock and as such it is important to minimise mechanical damage in crops. Effective disease management including growing resistant varieties and implementing fungicide strategies will also reduce the risk of disease staining on grain that might be confused with field moulds.

While visual appraisal of grain was often not able to distinguish between disease staining from ascochyta blight, staining from field moulds or staining from weather damage the laboratory tests took 14 days for mould counts and 4 days for toxin tests. The possibility of rapid and accurate objective testing to distinguish between these factors at silo receivals needs to be investigated using methodologies such as image analysis, ELISA tests or DNA assays.

Acknowledgments This study was funded by South Australian Grains Industry Trust. Grain samples were collected by Sam Holmes of Holmes Farm Consulting and by staff of SARDI Clare New Variety Agronomy. Mould counts and associated mycotoxin tests were conducted by Symbioalliance, Eight Mile Plains, Queensland, Australia. Thanks to Natalie Dowsett, SARDI, and Geoff Thomas, Thomas Project Services, for assistance with this manuscript.

\section{References}

Attitalla IH, Al-Ani LKT, Nasib MA, Balal IAA, Zakaria M, El-Maraghy SSM, Karim SMR (2010) Screening of fungi associated with commercial grains and animal feeds in Al-Bayda Governate, Libya. World Appl Sci J 9:746-656

Bottalico A, Perrone G (2002) Toxigenic Fusarium species and mycotoxins associated with head blight in small-grain cereals in Europe. Eur J Plant Pathol 108:611-624

Bureau of Meteorology (2011) Daily weather observations for South Australia. www.bom.gov.au accessed 20 April 2011

Cressey PJ (2009) Mycotoxin risk management in New Zealand and Australian food. World Mycotoxin J 2:113-118

DAFF (Department of Agriculture, Fisheries and Forestry) (2011) National residue survey residue monitoring. http://www.daff.gov.au/ agriculture-food/nrs/lab accessed 25 May 2011

El-Maraghy SSM (1988) Aflatoxins and fungal flora in lentil (Lens esculata L.). Mycopathologia 102:31-35

El-Nagarabi SAF, Elshafie AE (2000) Incidence of seed-borne fungi and aflatoxins in Sudanese lentil seeds. Mycopathologia 149:151156

FAO (Food and Agriculture Organization) (2004) Worldwide regulations for mycotoxins in food and feed in 2003. FAO Food and Nutrition Paper 81, Rome, Italy

Hawthorne W, Materne M, Davidson J, Lindbeck K, McMurray L, Brand J (2011) Lentil Disease management strategy. Disease 
management guide series PA2011\#13, Pulse Australia, Sydney, Australia

Husssain MA, Mukhtar T, Ul-Haque MI, Kayani MZ (2007) Mycoflora associated with lentil (Lens esculenta Moench) seeds from five localities of Punjab, Pakistan. Pak J Bot 39:903-906

Kaiser WJ (1992) Fungi associated with the seeds of commercial lentils from the U.S. Pacific Northwest. Plant Dis 76:605-610
Pulse Australia (2011) Industry submissions on pulse trading standards 2011/12 season. http://www.pulseaus.com.au/ receivalandtradingstandards. Accessed 20 April 2011

Rahim S, Dawar S, Tariq M (2010) Mycoflora associated with lentil (Lens culinaris L.) seeds of Pakistan. Pak J Bot 42:4345-4352

Sumar SP, Howard RJ (1983) Seed microflora of pulses in Alberta Canada. Seed Sci Technol 11:363-369 